\title{
Long-term monitoring of active stars
}

\section{VII. $\boldsymbol{U} B \boldsymbol{V}(\boldsymbol{R I})_{\mathrm{c}}$ photometry collected in March $1991^{\star, \star \star}$}

\author{
G. Cutispoto \\ Osservatorio Astrofisico di Catania, V.le A.Doria 6, I-95125 Catania, Italy \\ e-mail: gcutispoto@alpha4.ct.astro.it
}

Received April 21; accepted May 5, 1997

\begin{abstract}
In the framework of an extensive program focusing on the global properties and evolution of active stars, high-precision $U B V(R I)_{\text {c }}$ photometry of 19 selected stars, collected at the European Southern Observatory over the 15-31 March 1991 interval is presented. Significant evolution of the light curves, period variations and evidence for long-term variability of the global degree of spottedness are found. Most of the spectral classifications are discussed. A flare event was detected for the star HD $127535=\mathrm{V} 841$ Cen. These observations contribute to the establishment of a time-extended photometric database which can give important clues on topics such as the stability of spotted areas, differential rotation, solar-like cycles and the correlation between inhomogeneities at different atmospheric levels ${ }^{1}$.
\end{abstract}

Key words: stars: activity — stars: flare — stars: late-type - stars: variables - techniques: photometric — stars: HD 127535

\section{Introduction}

One of the most typical signatures of stellar activity is the presence of photospheric inhomogeneities, whose visibility, modulated by the stellar rotation, produces periodic or quasi-periodic light variation typically of the order of $0.1-0.2$ magnitudes in the $V$-band (cf. Rodonò 1992a,b and references therein). Multicolor photometry shows, in most cases, a reddening of the star at minimum

\footnotetext{
Send offprint requests to: G. Cutispoto

* based on data collected at the European Southern Observatory, La Silla, Chile.

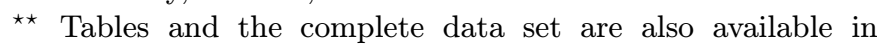
electronic form at the CDS via anonymous ftp to cdsarc.ustrasbg.fr or via http://cdsweb.u-strasbg.fr/Abstract.html

1 Figure 1 to 24 are only available on the on-line version of A\&A at http://www.ed-phys.fr
}

luminosity, thus supporting the cool starspot hypothesis. However, anticorrelation of the $U-B$ and $B-V$ color indices with respect to the $V$-band light modulation has been observed for some stars such as V711 Tau, TW Lep and UX Ari (Cutispoto 1992; Rodonò \& Cutispoto 1992; this paper). The orbital/photometric periods of active stars span from less than one day to several weeks and the photometric waves can undergo noticeable changes over time scales as short as few stellar rotations (cf. Figs. 12, 18 and 23 in Cutispoto 1995). Hence, in order to investigate the physical characteristics and evolution of spotted areas and the time scale of activity cycles, active stars must be observed systematically. This continuous monitoring program, already started at Catania Astrophysical Observatory in the early Sixties, is being carried out by using the 0.5 and $1.0 \mathrm{~m}$ telescopes of the European Southern Observatory (ESO, La Silla, Chile), the $0.25 \mathrm{~m}$ Automatic Photoelectric Telescope of Franklin \& Marshall College at Washington Camp. (AZ, U.S.A.) and the $0.8 \mathrm{~m}$ Automatic Photoelectric Telescope of Catania Astrophysical Observatory on Mt. Etna (Italy). It is aimed at establishing the time-extended database that is essential to investigate fundamental topics such as the evolution of spotted areas and spot lifetimes, the presence of photospheric solar-like activity cycles and differential rotation (see, among others, Cutispoto \& Rodonò 1992; Budding \& Zeilik 1995; Rodonò 1992a,b; Lanza et al. 1997), the temporal and/or spatial correlation between inhomogeneities at different atmospheric levels (Pagano et al. 1992, 1993; Pallavicini et al. 1993; Kürster 1996; Kürster et al. 1994, 1997; Catalano et al. 1996). This paper reports on data obtained by using the $0.5 \mathrm{~m}$ ESO telescope and is organized as follows: the details on the equipment, observations and reduction procedures are given in Sect. 2, the results and the discussion on individual stars are presented in Sect. 3. 
Table 1. Comparison $(c)$ and check $(c k)$ stars for each program star $(v)$ and standard deviations $(\sigma)$ for the $v-c$ and $c k-c$ $V$-band differential magnitudes for each series of $\mathrm{N}$ nights

\begin{tabular}{|cl|l|l|c|c|c|}
\hline \multicolumn{2}{|c|}{$\begin{array}{c}\text { Program star } \\
(v)\end{array}$} & \multicolumn{1}{c|}{$(c)$} & \multicolumn{1}{c|}{$(c k)$} & $\begin{array}{c}\sigma \\
(v-c)\end{array}$ & $\mathrm{N}$ \\
\hline HD 32918 & $=$ YY Men & HD 33763 & HD 33747 & 54 & 4 & 13 \\
HD 36705 & $=$ AB Dor & HD 35230 & HD 36316 & 17 & 6 & 11 \\
HD 37847 & $=$ TW Lep & HD 37653 & SAO 170680 & 41 & 4 & 16 \\
HD 39917 & $=$ SZ Pic & HD 39901 & HD 39962 & 38 & 5 & 13 \\
HD 61245 & $=$ V344 Pup & HD 61390 & HD 61333 & 22 & 5 & 12 \\
HD 81410 & $=$ IL Hya & HD 81904 & HD 80991 & 46 & 4 & 14 \\
HD 82558 & $=$ LQ Hya & HD 82508 & HD 82447 & 25 & 4 & 12 \\
HD 86005 & HD 86034 & HD 85849 & 4 & 2 & 10 \\
HD 98712 & $=$ SZ Crt & HD 97977 & HD 98251 & 20 & 4 & 11 \\
HD 101309 & $=$ V829 Cen & HD 101679 & HD 101215 & 50 & 4 & 13 \\
HD 102077 & $=$ V838 Cen & HD 102076 & HD 102202 & 16 & 4 & 13 \\
HD 106225 & $=$ HU Vir & HD 106270 & HD 105796 & 72 & 4 & 16 \\
HD 119285 & $=$ V851 Cen & HD 119164 & HD 119076 & 14 & 2 & 13 \\
HD 127535 & $=$ V841 Cen & HD 128227 & HD 128618 & 33 & 4 & 17 \\
HD 136905 & $=$ GX Lib & HD 136480 & HD 137241 & 49 & 4 & 14 \\
HD 139084 & $=$ V343 Nor & HD 139070 & HD 138363 & 60 & 4 & 13 \\
HD 155555 & $=$ V824 Ara & HD 157427 & HD 154775 & 25 & 7 & 16 \\
HD 174429 & $=$ PZ Tel & HD 173560 & HD 176557 & 21 & 5 & 17 \\
HD 197481 & $=$ AU Mic & HD 197237 & HD 197339 & 15 & 8 & 9 \\
\hline
\end{tabular}

Table 2. Mean atmospheric extinction coefficients for La Silla site, obtained over the 15-31 Mar. 1991 period

\begin{tabular}{|c|c|c|c|c|c|}
\hline & $U$ & $B$ & $V$ & $R$ & $I$ \\
\hline Mag/airmass & .520 & .274 & .157 & .107 & .053 \\
\hline
\end{tabular}

\section{The observations}

The present observations were carried out at the European Southern Observatory (La Silla, Chile) over the 15-31 March 1991 period, by using the $0.5 \mathrm{~m}$ ESO telescope equipped with a single-channel photon-counting photometer, a thermoelectrically cooled Hamamatzu R-943/02 photomultiplier and standard ESO filters matching the $U B V(R I)_{\text {c }}$ system. In order to obtain accurate differential photometry, for each program star $(v)$ a comparison (c) and a check (ck) star were also observed (see Table 1). The measurement of a star consisted in the average of $10-151$-s integrations in each filter, according to the $U-B-V-R_{\mathrm{c}}-I_{\mathrm{c}}$ color sequence. A complete observation consisted in sequential $c-v-v-v-v-c k-c$ measurements. From these data, after accurate sky subtraction, four $v-c$ and one $c k-c$ differential magnitudes were computed; the four $v-c$ values were finally averaged to obtain one data point. The observations were corrected for atmospheric extinction and transformed into the standard $U B V(R I)_{\text {c }}$ system. The nightly atmospheric extinction coefficients were obtained by observing two standards of very different spectral types in the $1-2.5$ air mass range. Their mean values over the whole period are re- ported in Table 2. These coefficients were obtained few months before the June 1991 eruption of Mt. Pinatubo. Transformation coefficients were inferred by observing Eregion standard stars (Menzies et al. 1989). The typical error of the differential photometry is of the order of 0.005 magnitudes, with somewhat larger values (up to 0.01 magnitudes) in the $U$-band due to the low photon counting level. The standard deviations $(\sigma)$ for the $v-c$ and $c k-c$ mean differential $V$-band magnitudes obtained over $N$ nights are reported in Table 1 . The $V$ magnitudes and colors of the comparison and check stars were obtained via standard stars (Menzies \& Laing 1988; Menzies et al. 1989; Menzies et al. 1991) and are given in Table 3. For each program star the brighest $V$ magnitude and corresponding colors are listed in Table 4. Taking into account the accuracy of the standard stars data and the extinction and transformation errors, the typical accuracy of the absolute photometry in Tables 3 and 4 is of the order of 0.01 magnitudes, with somewhat larger values (up to 0.02 magnitudes) for $U-B$ colors.

\section{Results}

The present multicolor photometry has been used to investigate the light curve evolutions, to search for the presence of photospheric solar-like activity cycles and, using the method described by Cutispoto et al. (1996), to infer or further constrain the spectral type and luminosity class of program stars (see Table 4). Color indices of active stars have to be taken prudently when used for spectral 
Table 3. $V$ magnitude and colors for the $c$ and $c k$ stars derived from standard stars. Errors are of the order of 0.01 magnitudes. The symbol ":" denotes errors of the order of 0.02 magnitudes

\begin{tabular}{|l|c|l|c|c|c|}
\hline \multicolumn{1}{|c}{$c$ or $c k$} & $V$ & $U-B$ & $B-V$ & $V-R_{\mathrm{c}}$ & $V-I_{\mathrm{c}}$ \\
\hline HD 33747 & 8.76 & 0.72 & 0.98 & 0.53 & 1.03 \\
HD 33763 & 8.37 & 0.50 & 0.89 & 0.49 & 0.95 \\
HD 35230 & 7.58 & 0.47 & 0.88 & 0.48 & 0.93 \\
HD 36316 & 7.95 & 1.72 & 1.46 & 0.79 & 1.50 \\
HD 37653 & 8.24 & 0.50 & 0.89 & 0.48 & 0.93 \\
SAO 170680 & 8.97 & 0.91 & 1.04 & 0.54 & 1.01 \\
HD 39901 & 6.54 & 1.63 & 1.37 & 0.71 & 1.32 \\
HD 39962 & 7.97 & $-.06:$ & 0.40 & 0.26 & 0.50 \\
HD 61333 & 7.18 & $-.64:$ & -.11 & -.05 & -.09 \\
HD 61390 & 7.92 & 0.70 & 1.01 & 0.52 & 1.03 \\
HD 81904 & 8.02 & $0.72:$ & 0.98 & 0.50 & 0.98 \\
HD 80991 & 8.51 & $0.83:$ & 1.04 & 0.52 & 1.02 \\
HD 82477 & 6.11 & 1.18 & 1.18 & 0.61 & 1.17 \\
HD 82508 & 7.58 & 0.35 & 0.71 & 0.40 & 0.80 \\
HD 85849 & 8.52 & 1.07 & 1.14 & 0.58 & 1.12 \\
HD 86034 & 7.89 & 1.32 & 1.26 & 0.63 & 1.20 \\
HD 97977 & 8.85 & $1.66:$ & 1.42 & 0.75 & 1.42 \\
HD 98251 & 9.21 & $1.47:$ & 1.31 & 0.69 & 1.28 \\
HD 101215 & 7.79 & 0.07 & 0.15 & 0.10 & 0.20 \\
HD 101679 & 8.12 & 0.79 & 1.09 & 0.59 & 1.15 \\
HD 102076 & 7.10 & 0.75 & 1.00 & 0.51 & 0.98 \\
HD 102202 & 8.86 & 0.19 & 0.67 & 0.38 & 0.73 \\
HD 105796 & 8.06 & 0.93 & 1.06 & 0.55 & 1.04 \\
HD 106270 & 7.58 & 0.29 & 0.72 & 0.40 & 0.77 \\
HD 119076 & 6.87 & 0.88 & 1.15 & 0.60 & 1.15 \\
HD 119164 & 7.20 & 1.14 & 1.29 & 0.64 & 1.22 \\
HD 128227 & 8.33 & 0.82 & 1.07 & 0.57 & 1.10 \\
HD 128618 & 8.03 & $1.63:$ & 1.46 & 0.77 & 1.47 \\
HD 136480 & 7.35 & $1.12:$ & 1.16 & 0.60 & 1.15 \\
HD 137241 & 7.36 & $1.10:$ & 1.14 & 0.59 & 1.11 \\
HD 138363 & 7.12 & 0.95 & 1.14 & 0.61 & 1.15 \\
HD 139070 & 8.71 & 0.80 & 1.12 & 0.60 & 1.16 \\
HD 154775 & 7.60 & 1.97 & 1.59 & 0.87 & 1.75 \\
HD 157427 & 7.41 & 1.69 & 1.50 & 0.81 & 1.55 \\
HD 173560 & 8.72 & $0.11:$ & 0.64 & 0.36 & 0.70 \\
HD 176557 & 7.19 & $1.76:$ & 1.48 & 0.78 & 1.47 \\
HD 197237 & 9.02 & $0.83:$ & 0.98 & 0.51 & 0.96 \\
HD 197339 & 7.39 & $1.44:$ & $1.29:$ & 0.66 & 1.23 \\
\hline
\end{tabular}

classification, as the presence of activity phenomena can modify them by an unknown amount. In a recent paper Amado \& Byrne (1997) have investigated the effects of stellar activity on photometric colors, showing that active stars have a bluer $U-B$ index compared to non-active stars of the same spectral type. These new data have been now taken into account computing the spectral type listed in Table 4. In most cases the spectral types obtained from color indices agree very well with the spectral classification, when available, as also shown for a larger sample of late-type stars by Metanomski et al. (1997).

The results for the individual stars are now discussed.

HD $32918=$ YY Men is an active giant with the typical characteristics of FK Com stars (see Cutispoto et al. 1992 and references therein). It has shown two of the most intense and longest duration radio flares (Slee et al. 1987a; Bunton et al. 1989) and probably the longest optical flare ever recorded for any class of active stars (Cutispoto et al. 1992). The data obtained over the 15-29 Mar. interval are shown in Fig. 1, where phases are reckoned from the 9.5476-day photometric period computed by Collier (1982a). The asymmetric $V$-band light curve is single-peaked and its maximum results about 0.045 magnitudes fainter than the brighest values observed to date (cf. Fig. 7 in Cutispoto \& Leto 1997). Remarkable color variations, well correlated with the $V$-band modulation, show the star to be redder at light minimum.

HD $36705=$ AB Dor is one of the most studied active stars as can be inferred from the papers listed in the SIMBAD database (see, among the most recent, Mewe et al. 1996; Rucinski et al. 1995; Cutispoto 1995; Unruh et al. 1995; Kürster et al. 1994, 1997 and references therein). The data obtained over the 15-28 Mar. interval are plotted in Fig. 2, where phases are reckoned from the 0.51423 day photometric period computed by Pakull (1981). The low amplitude $V$-band light curve exhibits two close maxima at phases $\sim 0.26$ and $\sim 0.49$, respectively. The weak color variations, better visible for the $U-B$ and $V-I$ indices, are in phase with the $V$ modulation. However, it is interesting to note that the primary maximum for the $U-B$ color curve corresponds to the maximum in the $V$ curve at phase $\sim 0.49$, while the primary maximum for the $V-I$ color curve corresponds to the phase $\sim 0.26$ maximum in the $V$ curve. Finally, the shape of the light curve is quite different from that observed in February 1991 by Anders et al. (1992), when only one maximum was present and the peak-to-peak amplitude was smaller. The colors agree fairly well with those of an active $\mathrm{K} 0 \mathrm{IV} / \mathrm{V}$ star. From the $v \sin i$ value computed by Randich et al. (1993) a minimum stellar radius of about $0.86 R_{\odot}$ is inferred. This is in agreement with the conclusion, reached by Rucinski (1985) and Innis et al. (1986), that HD 36705 has not arrived to the mean sequence yet.

HD $37847=$ TW Lep is a SB2 spectroscopic binary with an orbital period of 28.344 days (Balona 1987) that has been recentely observed by several authors (see, among others, Strassmeier \& Fekel 1990; Strassmeier et al. 1993a; Fox et al. 1994; Cutispoto 1995 and references therein). It was detected by the ROSAT satellite during the all-sky EUV survey (Pye et al. 1995). The photometric variability, with a period of 28.22 days, was first reported by Henry et al. (1982). The observations presented here were carried out over the 15-31 Mar. interval with the $0.5 \mathrm{~m}$ ESO. Three additional points were obtained with the $1.0 \mathrm{~m}$ ESO on 9, 10 and 12 April. The data are folded by using the 28.22-day photometric period and are shown in Fig. 3. The $V$-band light curve is single-peaked and the maximum luminosity is close to that observed during the 
Table 4. Maximum luminosity $\left(V_{\max }\right)$ and corresponding colors for the program stars and inferred spectral classification (Spectral Type)

\begin{tabular}{|c|c|c|c|c|c|c|c|}
\hline \multicolumn{2}{|c|}{ Program Star } & $V_{\max }$ & $U-B$ & $B-V$ & $V-R_{\mathrm{c}}$ & $V-I_{\mathrm{c}}$ & Spectral Type \\
\hline HD 32918 & $=$ YY Men & 7.98 & 0.67 & 1.04 & 0.59 & 1.14 & K1 III \\
\hline HD 36705 & $=\mathrm{AB}$ Dor & 6.89 & 0.37 & 0.83 & 0.49 & 0.97 & K0 IV/V (PMS) \\
\hline HD 37847 & $=\mathrm{TW}$ Lep & 7.35 & 0.76 & 1.09 & 0.60 & 1.16 & $\mathrm{~K} 2 / 3 \mathrm{III}+\mathrm{F} 6 \mathrm{IV}$ \\
\hline HD 39917 & $=\mathrm{SZ} \mathrm{Pic}$ & 7.85 & 0.31 & 0.81 & 0.47 & 0.91 & $\mathrm{~K} 0 / 1 \mathrm{IV}+\mathrm{G} 5 \mathrm{IV}$ \\
\hline HD 61245 & $=$ V344 Pup & 6.85 & 0.80 & 1.04 & 0.55 & 1.05 & K1 III \\
\hline HD 81410 & $=\mathrm{IL} \mathrm{Hya}$ & 7.33 & 0.74 & 1.03 & 0.55 & 1.09 & K2 IV \\
\hline HD 82558 & $=\mathrm{LQ} \mathrm{Hya}$ & 7.81 & 0.56 & 0.91 & 0.52 & 1.02 & $\mathrm{~K} 2 \mathrm{~V}$ \\
\hline HD 86005 & & 7.18 & 1.01 & 1.31 & 0.69 & 1.34 & $\mathrm{~K} 4 \mathrm{III}+\mathrm{K} 1 / 2 \mathrm{III}$ \\
\hline HD $98712 \mathrm{AB}$ & $=\mathrm{SZ}$ Crt & 8.60 & 1.21 & 1.33 & 0.86 & 1.65 & $\mathrm{~K} 7 \mathrm{~V}+\mathrm{M} 3 \mathrm{~V}$ \\
\hline HD $98712 \mathrm{~A}$ & & 8.74 & & 1.30 & 0.83 & 1.56 & $\mathrm{~K} 7 \mathrm{~V}$ \\
\hline HD 98712 B & & 11.25 & & 1.6 & 1.1 & 2.4 & M3 V \\
\hline HD 101309 & $=$ V829 Cen & 7.82 & 0.49 & 0.93 & 0.53 & 1.04 & $\mathrm{~K} 1 / 2 \mathrm{IV}+\mathrm{G} 5 \mathrm{~V}$ \\
\hline HD 102077 & $=\mathrm{V} 838$ Cen & 8.92 & 0.53 & 0.91 & 0.54 & 1.08 & $\mathrm{~K} 1 / 2 \mathrm{~V}+\mathrm{K} 2 \mathrm{~V}$ \\
\hline HD 106225 & $=\mathrm{HU}$ Vir & 8.59 & 0.58 & 0.98 & 0.57 & 1.13 & K1 IV/III \\
\hline HD 119285 & $=$ V851 Cen & 7.69 & 0.81 & 1.09 & 0.61 & 1.20 & $\mathrm{~K} 2 / 3 \mathrm{IV}$ \\
\hline HD 127535 & $=$ V841 Cen & 8.55 & 0.82 & 1.08 & 0.61 & 1.18 & $\mathrm{~K} 2 / 3 \mathrm{IV}$ \\
\hline HD 136905 & $=\mathrm{GX} \mathrm{Lib}$ & 7.30 & 0.73 & 1.03 & 0.57 & 1.10 & $\mathrm{~K} 1 \mathrm{III}+\mathrm{G} 5 \mathrm{IV}$ \\
\hline HD 139084 & $=$ V343 Nor & 8.06 & 0.37 & 0.82 & 0.48 & 0.93 & K0 IV/V (PMS) \\
\hline HD 155555 & $=\mathrm{V} 825$ Ara & 6.87 & 0.38 & 0.82 & 0.49 & 0.93 & G5 IV + K0 IV (PMS) \\
\hline HD 174429 & $=\mathrm{PZ}$ Tel & 8.44 & 0.31 & 0.78 & 0.45 & 0.87 & G9 IV/V (PMS) \\
\hline HD 197481 & $=\mathrm{AU} \mathrm{Mic}$ & 8.73 & 1.12 & 1.45 & 0.94 & 2.06 & M1 V \\
\hline
\end{tabular}

Jan.-Mar. 1989 period (Cutispoto 1992). The behavior of the color curves is rather peculiar. With respect to the $V$-band modulation the $U-B$ and the $B-V$ color curves are clearly anticorrelated, while the $V-R$ and $V-I$ color curves are well correlated. This behavior is qualitatively the same exibited by TW Lep during Jan.-Mar. 1989 (Cutispoto 1992), but is remarkably different from that observed in Dec. 1989 (Cutispoto 1995) when the $U-B$ and $B-V$ color curves appeared rather flat. Strassmeier $\&$ Fekel (1990) report the spectral classification K2 III + F6 IV. The observed colors are reproduced very well by those of a K2/3 III + F6 IV system with an active K-type giant component.

HD $39917=$ SZ Pic is an SB2 spectroscopic binary, with an orbital period of about 4.96 days (Kürster 1997), showing strong Ca II H\&K emission from both components (Andersen et al. 1980; Henry et al. 1996), very low Li abundance (Pallavicini et al. 1992; Randich et al. 1993) and photometric variability (Andersen et al. 1980; Bell et al. 1983; Cutispoto 1995). Moreover, it has been detected by the ROSAT satellite and by the VLA (Fox et al. 1994). It was observed over the 15-31 Mar. interval and these data, folded by using the 4.905-day period inferred by Cutispoto (1995), are presented in Fig. 4. The $V$-band light curve, though incomplete, appears quite different from the one obtained in Dec. 1989 and the color variations are marginal. Assuming both components to be active evolved stars, see Cutispoto (1995) and references therein, the spectral classification $\mathrm{K} 0 / 1 \mathrm{IV}+\mathrm{G} 5 \mathrm{IV}$ gives the best fit of the observed colors.

HD $61245=$ V 344 Pup is an SB1 spectroscopic binary that shows weak Ca II $\mathrm{H} \& \mathrm{~K}$ emission lines and $\mathrm{H} \alpha$ absorption (Bopp \& Hearnshaw 1983). The optical variability was discovered by Lloyd-Evans \& Koen (1987), further photometric observations were acquired by Cutispoto $(1992,1995)$. The data presented in this paper, obtained over the 15-27 Mar. interval, are shown in Fig. 5. The 11.761-day orbital period inferred by Balona (1987) has been used for phase computation. The $V$-band light curve is double-peaked while the weak color variations appear to be in phase with the $V$-band modulation. A remarkable evolution of the HD 61245 light curve is clear when comparing the present data with previuos observations (Cutispoto 1992, 1995). HD 61245 was classified as K1 III and as K2 III by Bopp \& Hearnshaw (1983) and Houk (1978), respectively. In order to fit the peculiar $U-B$ color Cutispoto $(1992,1995)$ had to assume the presence of an F8 IV companion. From the results of Amado \& Byrne (1997) it is now clear that the peculiar value of the $U-B$ can be entirely ascribed to the presence of photospheric activity. The observed colors are indeed very well matched by those of a single active K1 III star.

HD 81410 = IL Hya is an SB1 spectroscopic binary that has been observed by several authors at different wavelenghts (see Strassmeier et al. 1993a; Cutispoto 
1993, 1995 and references therein). More recently, it has been detected by the ROSAT and EUVE satellites (Pye et al. 1995; Bowyer et al. 1996; Mitrou et al. 1997). The data presented in this paper were obtained over the 15-28 Mar. interval and are shown in Fig. 6, where phases are reckoned from the 12.86833-day orbital period computed by Raveendran et al. (1982). The $V$-band light curve is double-peaked and very clear color variations, showing the star redder at both light minima, are present. Comparing the present data with those obtained about 15 months earlier (Cutispoto 1995) it is clear that the photospheric spotted regions have undergone a remarkable evolution. The collection of the available photometry of IL Hya, shown in Fig. 7, is indicative of the presence of long-term variability of the global degree of spottedness. The data presented in this paper show HD 81410 to be close to the maximum luminosity observed in late 1989. Quite different spectral classifications of IL Hya are reported in the literature. The radius has been computed in the $5.5-5.9 R_{\odot}$ range (see discussion in Cutispoto 1993, 1995). In order to fit the peculiar $U-B$ color, Cutispoto (1995) assumes the presence of a G8 V companion. From the computation of Amado \& Byrne (1997) it is now clear that the peculiar $U-B$ can be entirely ascribed to the presence of photosperic activity. The observed colors are indeed very well matched with those of a single and active K2 IV or K0/1 III star. The subgiant classification, that agrees better with the results obtained by Houk \& SmithMoore (1988) and Eggen (1973), implies a value for the inclination angle close to 90 degrees, that is consistent with the very large amplitudes of the light curve observed for this star in the past (cf. Fig. 7).

HD 82558 = LQ Hya is a very active rapidly rotating single star. It has been recently observed at different wavelengths by several authors and classified as a very young or even as a PMS star (see Vilhu et al. 1991; Strassmeier et al. 1990; Fekel et al. 1986a and references therein). A very good photometric coverage has been obtained from late 1983 (see Jetsu 1993; Strassmeier et al. 1993b; Cutispoto 1996; Alekseev \& Gershberg 1996 and references therein). HD 82558 has been detected by the ROSAT (Pye et al. 1995) and EUVE (Bowyer et al. 1996) satellites. The data obtained over the 15-28 Mar. interval are shown in Fig. 8, where phases have been reckoned from the 1.5978-day photometric period computed by Strassmeier \& Hall (1988). The light curve is singlepeaked and the color variation appears in phase with the $V$-band modulation, showing the star redder at light mimimum. The colors are consistent with those of an active K2 V star.

HD 86005 was reported as a possible very active star by Bopp \& Hearnshaw (1983) because of $\mathrm{H} \alpha$ and Ca II $\mathrm{H} \& \mathrm{~K}$ emission lines and by Verma et al. (1983) because of a strong infrared excess. Scott et al. (1992) gave ev- idence for optical variability with a period of about 89 days. The present observations were obtained over the 15 25 Mar. interval, so that they cover only a small part of the light curve. The mean magnitude in the $V$-band results $7.187 \pm 0.004$, with the maximum and minimum luminosity differing in 0.014 magnitudes. Houk (1978) classified HD 86005 as K2 III and noticed the possibility that the star is a close double. Pallavicini et al. (1992) and Randich et al. (1993) reported a very low $\mathrm{Li}$ abundance and a $v \sin i$ in the $25-28 \mathrm{~km} \mathrm{~s}^{-1}$ range. This leads to compute a minimum radius in the $44-49 R_{\odot}$ range, a value that appears too high even for a luminosity class III star. Actually, the power spectrum presented by Scott et al. (1992) shows a significant second armonic, that corresponds to a period of 44.57 days, i.e. to a stellar radius in the $22-25 R_{\odot}$ range. Moreover, the lines profiles in the Li I $6708 \AA$ region are consistent with the star being a close binary (Randich 1997). In this case the high value of the $v \sin i$ could be due to a partial merging of the lines of the two components. There are several spectral combinations that can reproduce the observed colors of HD 86005, but only the systems K4 III + K1/2 III and $\mathrm{K} 4 \mathrm{III}+\mathrm{G} 3 \mathrm{IV} / \mathrm{III}$ could give origin to a composite spectrum.

HD $98712=$ SZ Crt is the visual binary ADS 8138 . The primary component shows a variable $\mathrm{H} \alpha$ that has been observed sporadically in emission (Torres et al. 1985; Bopp 1987). The secondary component shows strong $\mathrm{H} \alpha$ emission (Torres et al. 1985). The presence of optical variability, with a photometric period of 11.58 days, was first reported by Torres et al. (1985), further observations have been obtained by Cutispoto (1993, 1996). The observations presented in this paper were obtained over the 1627 Mar. interval and are shown in Fig. 9, where phases have been reckoned from the 11.58-day period computed by Torres et al. (1985). Due to the angular distance of about 5.1 arcsec both components were always observed simultaneously at the $0.5 \mathrm{~m}$ ESO. The light curve is singlepeaked and shows the largest amplitude and the brightest maximum luminosity observed to date for this star. The $V-I$ index shows clear correlation with the $V$-band modulation. Separate magnitudes for the two components (see Table 4) were obtained with the $1.0 \mathrm{~m}$ ESO telescope on the night of 10 April 1991.

HD $101309=$ V 829 Cen is a SB2 system with an orbital period of 11.71 days (Balona 1987) that shows Ca II $\mathrm{H} \& \mathrm{~K}$ and $\mathrm{H} \alpha$ emission lines (Collier et al. 1982) and has been detected during a microwave survey of active stars (Slee et al. 1987b). A rather high Li abundance in both components has been reported by Randich et al. (1993). The photometric variability, discovered by Lloyd Evans \& Koen (1987), has been later investigated by Collier Cameron (1987) and by Cutispoto (1993, 1996). The present observations were collected over the 15-28 Mar. 
interval and are presented in Fig. 10, where the 11.65-day photometric period inferred by Cutispoto (1993) was used for phase computation. The light curve is single-peaked and clear $V-I$ color variation in phase with $V$-band modulation are present; the $U-B$ data are more scattered but they seem to be anticorrelated with the $V$-band data. A collection of the available photometric data of HD 101309, shown in Fig. 11, gives evidence for sizeable changes of the global degree of spottedness. In particular, during the time interval covered by the present observations HD 101309 was seen at the brightest luminosity ever observed. The colous are consistent with those of a $\mathrm{K} 1 / 2 \mathrm{IV}+\mathrm{G} 5 \mathrm{~V}$ active system, in very good agreement with the spectral classification inferred by Collier et al. (1982).

HD $102077=\mathrm{V} 838 \mathrm{Cen}$ is a close visual binary (sep. $\simeq 0.2$ arcsec) consisting of two almost identical components (Turon et al. 1992) that show moderate $\mathrm{Ca}$ II $\mathrm{H} \& \mathrm{~K}$ emission lines and a filled-in $\mathrm{H} \alpha$ line (Bopp et al. 1986). Its photometric variability was first reported by Udalski \& Geyer (1985a). Further photometry has been collected by Bopp et al. (1986), Cutispoto $(1990,1993,1996)$ and by Anders et al. (1991). The observations presented in Fig. 12 were obtained over the 1529 Mar. interval. Phases have been computed by using the $1.848 \pm 0.023$ day photometric period inferred from a Fourier analysis of VRI data. The light curve is doublepeaked and the weak color variations show the system to be redder at light minimum. Houk (1978) lists a K0/1Vp spectral type for the composite spectrum. The observed colors fit those of a $\mathrm{K} 1 / 2 \mathrm{~V}+\mathrm{K} 2 \mathrm{~V}$ system with active components. For such a system the luminosity difference between the two components $\left(\Delta M_{v}=0.12\right)$ results in agreement with the observations (Turon et al. 1992).

HD $106225=$ HU Vir is a SB1 spectroscopic binary with an orbital period of 10.38758 days (Strassmeier 1994) showing very strong Ca II H\&K emission lines (Montes et al. 1996) and a variable $\mathrm{H} \alpha$ line (Strassmeier 1994). It has been detected in a radio survey of $\mathrm{RS}$ Cvn-type systems (Morris \& Mutel 1988; Drake et al. 1989) and a huge X-ray flare, lasting about two days, was observed by the ROSAT satellite (Endl et al. 1995). For a detailed study of the physical parameters of HD 106225 see Strassmeier (1994). The optical variability has been studied by several authors in recent years (see Strassmeier et al. 1997, 1993a; Cutispoto 1996; Hall \& Henry 1992 and references therein). The observations presented here were obtained over the 15-30 Mar. interval and are shown in Fig. 13, where phases are computed by using the 10.353-day photometric period inferred by Strassmeier et al. (1997). The light curve is double-peaked and large amplitude color variations, showing the star redder at both light minima, are present. The colors agree well with those of an active K1 IV or K1 IV/III star, in good agreement with the results of Strassmaier (1994).
Photometric periods ranging from 10.102 to 10.6 days (Strassmeier et al. 1997; Fekel et al. 1986b) and $v \sin i$ values in the $25-28 \mathrm{~km} \mathrm{~s}^{-1}$ range (Randich et al. 1994; Fekel et al. 1986b) have been observed for HD 106225. Moreover, for the inclination angle " $i$ ", Strassmeier (1994) deduced values in the $52-78$ degrees range. These results lead to a stellar radius in the $5.1-7.4 R_{\odot}$ range, in better agreement with a luminosity class IV/III.

HD $119285=$ V 851 Cen is an SB1 system with an orbital period of 11.9886 days (Saar et al. 1990) that has been studied by several authors in recent years (see Saar et al. 1990; Cutispoto 1993, 1996 and references therein). It has been also detected by ROSAT satellite (Pye et al. 1995) and low Li abundance has been reported by Randich et al. (1993). The optical variability was first discovered by Udalki \& Geyer (1984) and by Lloyd Evans \& Koen (1987). The data presented in Fig. 14 were collected over the 15-27 Mar. interval. The 12.05day photometric period reported by Lloyd Evans \& Koen (1987) was used for phase computation. The light curve is single-peaked and the low-amplitude color variations are in phase with the $V$-band modulation. From the collection of the available photometry of HD 119285 shown in Fig. 15, it seems that the $V$-band mean stellar luminosity is decreasing after the maximum observed in early 1990 (Cutispoto 1996). A luminosity class IV or IV/V was deduced by Cutispoto (1996) for the primary component of HD 119285. The colors are consistent with those of an active K2/3 IV star, in good agreement with the K2 IV classification reported by Saar et al. (1990).

HD $127535=$ V 841 Cen is an SB1 system, with an orbital period of 5.998 days (Collier 1982b), showing strong $\mathrm{Ca}$ II $\mathrm{H} \& \mathrm{~K}$ and $\mathrm{H} \alpha$ emission lines (Houk \& Cowley 1975; Weiler \& Stencel 1979; Collier et al. 1982). It was detected at radio (Innis et al. 1985; Slee \& Stewart 1989) and EUV (Pye et al. 1995) wavelengths. Low Li abundance was reported by Randich et al. (1993). The photometric variability was discovered by Collier (1982a) and Udalski \& Geyer (1984). The observations obtained over the 15-31 Mar. interval are shown in Fig. 16, where the 5.929-day photometric period computed by Cutispoto (1990) was used for phase computation. The light curve is quite peculiar, as it shows a well pronounced minimum at about phase 0.2 while it is almost flat in the $0.35-1.0$ interval. The low amplitude color variations show the star redder at light mimimum. Moreover, it is clear the presence of a flare event lasting at least one day. HD 127535 was flaring the first and second night of observations, as can be easily inferred from the color curves. There was no evidence of flare activity during any of the other 15 nights, that span over almost three complete rotational cicles. The length of the observed flare cannot be estimated from the present data. The collection of the available photometry of HD 127535 , shown in Fig. 17, confirms the occurrence of a minimum 
luminosity during the 1985-87 interval, while the most recent data (Strassmeier et al. 1994a) indicate a much lower degree of spottedness. Cutispoto (1996) inferred a luminosity class IV for the primary component. The colors are consistent with those of an active K2/3 IV star, in reasonable agreement with the K1 IV spectral classification given by Collier et al. (1982).

HD $136905=\mathbf{G X}$ Lib is an SB1 binary with an orbital period of 11.13448 days (Kaye et al. 1995). It shows moderate $\mathrm{Ca}$ II $\mathrm{H} \& \mathrm{~K}$ emission (Bidelman \& MacConnell 1973; Strassmeier et al. 1994b), strong $\mathrm{H} \alpha$ absorption (Eker et al. 1995) and very low Li abundance (Randich et al. 1993). It was detected by the Einstein (Fleming et al. 1989; Gioia et al. 1990) and by the ROSAT (Dempsey et al. 1993) satellites. Its photometric variability is thought to be mainly due to the ellipticity effect, where distortion due to starspots is superimposed (Burke et al. 1982; Cutispoto 1993, 1996; Kaye et al. 1995 and references therein). In this paper the observations obtained over the 15-29 Mar. interval are presented. The data are shown in Fig. 18, where the 11.13448-day spectroscopic period deduced by Kaye et al. (1995) was used for phase computation. The light curve is double-peaked and clear color variations showing the star redder at minimum luminosity are present. Comparing these data with previous observations the evolution of the light curve appears evident, thus confirming the presence of evolving spotted regions. Moreover, from the collection of the available photometry of HD 136905, shown in Fig. 19, a weak variation of the mean stellar brightness can be inferred. In particular, the present observations show the faintest stellar luminosity ever observed for this star. From the $v \sin i$ values computed by Randich et al. (1993) and by Favata et al. (1995) the minimum stellar radius results in the $7.7-9.7 R_{\odot}$ range. The spectral classification of $\mathrm{HD} 136905$ is given as K1 III $+[\mathrm{G}-\mathrm{K} \mathrm{V}]$ by Strassmeier et al. (1993a) and as K1 III + F by Bidelman \& MacConnell (1973). The two best fits of the observed colors are obtained by assuming K1 III + G5 IV or $\mathrm{K} 1 / 2 \mathrm{III}+\mathrm{G} 0 \mathrm{IV}$ systems with active components.

HD $139084=$ V 343 Nor is a very active single (Balona 1987) star belonging to the Pleiades group (Anders et al. 1991). It shows Ca II H\&K emission (Bidelman \& MacConnell 1973) and a filled $\mathrm{H} \alpha$ line (Bopp \& Hearnshaw 1983). A very high $\mathrm{Li}$ abundance was observed by Anders et al. (1991) and Randich et al. (1993). HD 139084 was detected during microwave (Slee et al. 1987b) and EUV (Pye et al. 1995; Bowyer et al. 1996) surveys. The optical variability was discovered by Udalski \& Geyer (1985b). The observations carried out over the 1528 Mar. interval are presented in Fig. 20, where phases have been reckoned from the $4.24 \pm 0.08$ day photometric period obtained from a Fourier analysis of the VRI data. The light curve is single-peaked and the very strong color variations show the star redder at light minimum.
An active $\mathrm{K} 0 \mathrm{IV} / \mathrm{V}$ star fits well the observed colors, in agreement with the minimum stellar radius that results in the $1.33-1.53 R_{\odot}$ range (Cutispoto 1996). This could imply, taking also into account the very high $\mathrm{Li}$ abundance, that HD 139084 has not arrived to the main sequence yet.

HD $155555=$ V 824 Ara is a rapidly rotating SB2 binary that has been classified as a pre-main sequence star by Pasquini et al. (1991) and by Martin \& Brandner (1995). Detection at EUV wavelengths has been obtained by the ROSAT (Pye et al. 1995) and EUVE (Bowyer et al. 1996) satellites. Photometric observations have been carried out from late 1979 by several authors (see Cutispoto \& Leto 1997; Cutispoto 1996 and references therein). The observations presented in Fig. 21 were acquired over the 1531 Mar. interval. Any light contribution from the M-type optical companion LDS587 B, that lies 32.6 arcsec apart, was avoided. Phases have been reckoned from the $1.694 \pm$ 0.010 day photometric period computed by a Fourier analysis of the $V R I$ data. The light curve is rather complicated and has a different shape and a smaller amplitude compared to the light curve obtained about six months before (Cutispoto \& Leto 1997). The low-amplitude color variations are in phase with the $V$-band modulation and show the system to be redder at minimum luminosity.

HD $\mathbf{1 7 4 4 2 9}=\mathbf{P Z}$ Tel is a single (Balona 1987; Innis et al. 1988) rapidly rotating active star that has been classified as a very young or as a pre-main sequence object (Innis et al. 1986). It shows a filled in $\mathrm{H} \alpha$ (Innis et al. 1988) and emission Ca II H\&K lines (Bidelman \& MacConnell 1973; Houk 1978; Innis et al. 1988). HD 174429 has been detected during a microwave survey of southern active stars (Slee et al. 1987b) and shows a very high Li abundance (Randich et al. 1993). The optical variability was discovered by Coates et al. (1980), further photometric data can be found in Bopp et al. (1986), Innis et al. (1990) and Cutispoto \& Leto (1997). The observations presented in Fig. 22 were obtained over the 15-31 Mar. interval, phases have been computed by using the $0.9447 \pm 0.0044$ day photometric period inferred from a Fourier analysis of the VRI data. The light curve is double-peaked, weak color variations, showing the star redder at the primary minimum, are present. The maximum luminosity is the same obtained about six months before (Cutispoto \& Leto 1997), but the light curve has a larger amplitude and a quite different shape. Houk (1978) classified PZ Tel as a K0 Vp and the observed colors are indeed in good agreement with those of an active G9/K0 dwarf or G9 IV/V stars. Randich et al. (1993) computed a $v \sin i$ of the order of $70 \mathrm{~km} \mathrm{~s}^{-1}$, which leads to infer a minimum stellar radius of about $1.31 R_{\odot}$. This radius is too high for a luminosity class $\mathrm{V}$ star, confirming that HD 174429 is probably still approaching the main sequence. 
HD $197481=$ AU Mic is a flare star that is also a member of the BY Dra-type variables. It shows the typical spectral features indicative of the presence of an active atmosphere (Linsky et al. 1982; Quin et al. 1993; Houdebine \& Doyle 1994; Monsignori-Fossi et al. 1996 and references therein). X-ray and EUV emission has been revealed by the Einstein (Golub 1983), ROSAT (Pye et al. 1995) and EUVE (Bowyer et al. 1996) satellites. The optical variability was discovered by Torres et al. (1972). The observations presented here, obtained over the 20-31 Mar. interval, are shown in Fig. 23, where phases are reckoned from the 4.865-day photometric period computed by Torres et al. (1972). The light curve is double-peaked and the $V-R$ and $V-I$ colors show the star to be redder at light minima. The $U-B$ and $B-V$ are not well correlated with the $V$-band modulation, a circumstance that could be due to the presence of micro-flaring activity. From the collection of the available photometry of HD 197481 shown in Fig. 24, in which remarkable variations of the mean $V$ magnitude and light curve amplitude can be seen, it is clear that sizeable modifications of the spotted areas and spot distribution do occur. The colors are consistent with the M1 Ve spectral classification reported by Houk (1982).

Acknowledgements. Stellar activity research at Catania Astrophysical Observatory is supported by the Italian "Ministry for Universities and Research" (MURST) and the "National Research Council" (Gruppo Nazionale di Astronomia) that are gratefully acknowledged. The author thank Ms. L. Santagati for revising the manuscript. This research has made use of the SIMBAD database, operated at CDS, Strasbourg, France. A special thank is also due to the ESO staff for the collaboration and technical support during the observations.

\section{References}

Alekseev I. Yu., Gershberg R.E., 1996, Ap 39, 33

Amado P.J., Byrne P.B., 1997, A\&A 319, 967

Anders G.J., Innis J.L., Coates D.W., Thompson K., 1991, MNRAS 252, 408

Anders G.J., Coates D.W., Thompson K., 1992, PASAu 10, 33

Andersen J. Nordstrom B., Olsen E.H., 1980, IAU Comm. 27, Inf. Bull. Var. Stars No. 1821

Balona L.A., 1987, S. Afr. Astron. Obs. Circ. 11, 1

Bell B.M., Hall D.S., Marcialis R.L., 1983, IAU Comm. 27, Inf. Bull. Var. Stars No. 2272

Bidelman W.P., MacConnell D.J., 1973, AJ 78, 687

Bopp B.W., 1987, ApJ 317, 781

Bopp B.W., Hearnshaw J.B., 1983, ApJ 267, 653

Bopp B.W., Africano J., Quigley R., 1986, AJ 92, 1409

Bowyer S., Lampton M., Lewis J., et al., 1996, ApJS 102, 129

Budding E., Zeilik M., 1995, Ap\&SS 232, 355

Bunton J.D., Large M.I., Slee O.B., et al., 1989, Proc. ASA 8, 127

Burke E.W., Baker J.E., Fekel F.C., Hall D.S., Henry G.W., 1982, IAU Comm. 27, Inf. Bull. Var. Stars No. 2951

Catalano S., Rodonò M., Frasca A., Cutispoto G., 1996, in "Stellar Surface Structure", IAU Symp. 176, Strassmeier
K.G. \& Linsky J.L. (eds.). Kluwer Academic Publishers, p. 403

Coates D.W., Halprin L., Sartori P., Thompson K., 1980, IAU Comm. 27 Inf. Bull. Var. Stars No. 1849

Collier A.C., 1982a, Southern Stars 30, 177

Collier A.C., 1982b, Ph.D. Thesis, University of Canterbury, New Zeland

Collier A.C., Haynes R.F., Slee O.B., Wright A.E., Hiller D.J., 1982, MNRAS 200, 869

Collier Cameron A., 1987, S. Afr. Astron. Obs. Circ. 11, 57

Cutispoto G., 1990, A\&AS 84, 397

Cutispoto G., 1992, A\&AS 95, 397

Cutispoto G., 1993, A\&AS 102, 655

Cutispoto G., 1995, A\&AS 111, 507

Cutispoto G., 1996, A\&AS 119, 281

Cutispoto G., Leto G., 1997, A\&AS 121, 369

Cutispoto G., Rodonó M., 1992, in "The Solar Cycle", Harvey K.L. (ed.) ASP Conf. Ser. 27, 465

Cutispoto G., Pagano I., Rodonò M., 1992, A\&A 263, L3

Cutispoto G., Tagliaferri G., Pallavicini R., Pasquini L., Rodonò M., 1996, A\&AS 115, 41

Dempsey R.C., Linsky J.E., Fleming T., Schmitt J.H.M.M., 1993, ApJS 86, 599

Drake S.A., Simon T., Linsky J.L., 1989, ApJS 71, 905

Endl M., Strassmeier K.G., Kürster M., 1995, in Poster Proceedings: "Stellar Surface Structure, IAU Symp. 176", Strassmeier K.G. (ed.). Univ. Vienna, p. 203

Eker Z., Hall D.S., Anderson C.M., 1985, ApJS 96, 581

Eggen O.J., 1973, PASP 85, 42

Favata F., Barbera M., Micela G., Sciortino S., 1995, A\&A 295,147

Fekel F.C., Bopp B.W., Africano J.L., et al., 1986a, AJ 92, 1150

Fekel F.C., Moffett T.J., Henry G.W., 1986b, ApJS 60, 551

Fleming T.A., Gioia I.M., Maccacaro T., 1989, AJ 98, 692

Fox D.C., Linsky J.L., Veale A., et al., 1994, A\&A 284, 91

Gioia I.M., Maccacaro T., Schild R.E., et al., 1990, ApJS 72, 567

Golub L., 1983, in "Activity in Red-Dwarf Stars", IAU Coll. 71, Byrne P.B. and Rodonò M. (eds.). D. Reidel Publ. Comp., p. 83

Hall D.S., Henry G.W., 1992, IAU Comm. 27 Inf. Bull. Var. Stars No. 3693

Henry G.W., Murray S., Hall D.S., 1982, IAU Comm. 27, Inf. Bull. Var. Stars No. 2214

Henry T.J., Soderblom D.R., Donahue R.A., Baliunas S.L., 1996, AJ 111, 439

Houdebine E.R., Doyle J.G., 1994, A\&A 289, 185

Houk N., 1978, "Michigan Catalogue of two dimensional spectral types for the HD stars", Vol. 2, Department of Astronomy University of Michigan, Ann Arbor

Houk N., 1982, "Michigan Catalogue of two dimensional spectral types for the HD stars", Vol. 3, Department of Astronomy University of Michigan, Ann Arbor

Houk N., Cowley A.P., 1975, "Michigan Catalogue of two dimensional spectral types for the HD stars", Vol. 1, Department of Astronomy University of Michigan, Ann Arbor

Houk N., Smith-Moore M., 1988, "Michigan Catalogue of two dimensional spectral types for the HD stars", Vol. 4, 
Department of Astronomy University of Michigan, Ann Arbor

Innis J.L., Coates D.W., Thompson K., et al., 1985, Proc. Astr. Soc. Austr. 6, 160

Innis J.L., Thompson K., Coates D.W., 1986, MNRAS 223, 183

Innis J.L., Coates D.W., Thompson K., 1988, MNRAS 233, 887

Innis J.L., Coates D.W., Thompson K., Lloyd Evans T., 1990, MNRAS 242, 306

Jetsu L., 1993, A\&A 276, 345

Kaye A.B., Hall D.S., Henry G.W., et al., 1995, AJ 109, 2177

Kürster M., 1996, in "Stellar Surface Structure" IAU Symp. 176, Strassmeier K.G. \& Linsky J.L. (eds.). Kluwer Academic Publishers, p. 477

Kürster M., 1997 (private communication)

Kürster M., Schmitt J.H.M.M., Cutispoto G., 1994, A\&A 289, 899

Kürster M., Schmitt J.H.M.M., Cutispoto G., Dennerl K., 1997, A\&A (in press)

Lanza A.F., Catalano S., Cutispoto G., Pagano I., Rodonò M., 1997, A\&A (submitted)

Linsky J.L., Bornmann P.L., Carpenter K.G., et al., 1982, ApJ 260,670

Lloyd Evans T., Koen M.C.J., 1987, S. Afr. Astron. Obs. Circ. 11,21

Malina R.F., Marshall H.L., Antia B., et al., 1994, AJ 107, 751

Martin E.L., Brandner W., 1995, A\&A 294, 744

Menzies J.W., Laing J.D., 1988, MNRAS 231, 1047

Menzies J.W., Cousins A.W.J., Banfiels R.M., Laing J.D., 1989, S. Afr. Astr. Obs. Circ. 13, 1

Menzies J.W., Marang F., Laing J.D., Coulson I.M., Engelbrecht C.A., 1991, MNRAS 248, 642

Metanomski A.D.F., Pasquini L., Krautter J., Cutispoto G., Fleming T.A., 1997, A\&A (submitted)

Mewe R., Kaastra J.S., White S.M., Pallavicini R., 1996, A\&A 315,170

Mitrou C.K., Mathioudakis M., Doyle J.G., Antonopoulov E., 1997, A\&A 317, 776

Monsignori Fossi B.C., Landini M., Del Zanna G., Bowyer S., 1996, ApJ 466, 427

Montes D., Fernandez-Figueroa M.J., Cornide M., De Castro E., 1996, A\&A 312, 221

Morris D.H., Mutel R.L., 1988, AJ 95, 204

Pagano I., Rodonò M., Neff J.E., 1992, in "Surface Inhomeogeneities in Late-Type Stars. Byrne P.B. \& Mullan D.J. (eds.), Lecture Notes in Physics. Springer-Verlag, p. 315

Pagano I., Rodonò M., Cutispoto G., et al., 1993, in "Physics of Solar and Stellar Coronae", Linsky J.F. \& Serio S. (eds.). Kluwer Academic Publishers, p. 457

Pallavicini R., Randich S., Giampapa M.S., 1992, A\&A 253, 185

Pallavicini R., Cutispoto G., Randich S., Gratton R., 1993, A\&A 267, 145

Pakull M.W., 1981, A\&A 104, 33

Pasquini L., Cutispoto G., Gratton R., Mayor M., 1991, A\&A 248, 72

Pye J.P., McGale P.A., Allan D.J., et al., 1995, MNRAS 274, 1165
Quin D.A., Doyle J.G., Butler C.J., Byrne P.B., Swank J.H., 1993, A\&A 272, 477

Randich S., 1997 (private communication)

Randich S., Gratton R., Pallavicini R., 1993, A\&A 273, 194

Randich S., Giampapa M.S., Pallavicini R., 1994, A\&A 283, 893

Raveendran A.V., Mekkaden M.V., Mohin S., 1982, MNRAS 199, 707

Rodonò M., 1992a, in "Surface Inhomogeneities in Late-Type Stars" Byrne P.B. \& Mulland D.J. (eds.), Lecture Notes in Physics. Springer-Verlag, p. 201

Rodonò M., 1992b, in "Evolutionary Processes in Interacting Binary Stars", Proc. 151st IAU Sym., Kondo Y. et al. (eds.). Kluwer Academic Publishers, p. 71

Rodonò M., Cutispoto G., 1992, A\&AS 95, 55

Rucinski S.M., 1985, MNRAS 215, 591

Rucinski S.M., Mewe R., Kaastra J.S., Vilhu O., White S.M., 1995, ApJ 449, 900

Saar S.H., Nordström B., Andersen J., 1990, A\&A 235, 291

Scott T.R., Hearnshaw J.B., Watson R.D., Kilmartin P.M., Gilmore A.C., 1992, JA\&A 13, 279

Slee O.B., Steward R.T., 1989, MNRAS 236, 129

Slee O.B., Nelson G.J., Steward R.T., et al., 1987a, MNRAS 227,467

Slee O.B., Nelson G.J., Steward R.T., et al., 1987b, MNRAS 229,659

Strassmeier K.G., 1994, A\&A 281, 395

Strassmeier K.G., Fekel F.C., 1990, A\&A 230, 389

Strassmeier K.G., Hall D.S., 1988, ApJS 67, 453

Strassmeier K.G., Fekel F.C., Bopp B.W., Dempsey R.C., Henry G.W., 1990, ApJS 72, 191

Strassmeier K.G., Hall D.S., Fekel F.C., Scheck M., 1993a, A\&AS 100, 173

Strassmeier K.G. Rice J.B., Wehlau W.H., et al., 1993b, A\&A 268,671

Strassmeier K.G., Paunzen E., North P., 1994a, IAU Comm. 27, Inf. Bull. Var. Stars No. 4066

Strassmeier K.G., Handler G., Pauzen E., Rauth M., 1994b, A\&A 281, 855

Strassmeier K.G., Bartus J., Cutispoto G., Rodonò M., 1997, A\&AS (in press)

Torres C.A.O., Ferraz Mello S., Quast G.R., 1972, Astrophys. Lett. 11,13

Torres C.A.O., Busko I.C., Quast G.R., 1985, Rev. Mex. Astron. Astrofis. 10, 329

Turon C., Crèzè M., Egret D., et al., 1992, "The Hipparcos Input Catalogue", ESA SP-1136

Udalski A., Geyer E.H., 1984, IAU Comm. 27 Inf. Bull. Var. Stars No. 2525

Udalski A., Geyer E.H., 1985a, IAU Comm. 27 Inf. Bull. Var. Stars No. 2691

Udalski A., Geyer E.H., 1985b, IAU Comm. 27 Inf. Bull. Var. Stars No. 2692

Unruh Y.C., Collier Cameron A., Cutispoto G., 1995, MNRAS 277,1145

Verma R.P., Ghosh S.V., Iyengar K.V.K., et al., 1983, Ap\&SS 97, 161

Vilhu O., Gustafsson B., Walter F.M., 1991, A\&A 241, 167

Weiler E.J., Stencel R.E., 1979, AJ 84, 1372 